\section{LA FILOSOFÍA DE LA CIENCIA COMO PROGRAMA DE INVESTIGACIÓN EN ZUBIRI}

\author{
Esteban Vargas ${ }^{1}$ \\ Pontificia Universidad Católica de Valparaíso \\ esteban.vargas@pucv.cl
}

Cómo citar este artículo/Citation: Vargas, E. (2016). La filosofía de la ciencia como programa de investigación en Zubiri. Arbor, 192 (780): a338. doi: http://dx.doi.org/10.3989/ arbor.2016.780n4014

Recibido: 25 julio 2014. Aceptado: 01 septiembre 2015.

RESUMEN: En este escrito se intenta explicar qué es ciencia en Zubiri. Aunque Zubiri no dejó un libro específico sobre ciencia, intentamos meditar este tema siguiendo el análisis en tres momentos de la aprehensión de realidad que hace Zubiri en su libro Inteligencia sentiente. A partir de allí, proponemos que el concepto de ciencia debe analizarse en estos tres momentos, el primero de los cuales muestra que el conocimiento científico hunde sus raíces en la realidad aprehendida sentientemente. La ciencia suele pensarse como una actividad racional. Esto sin duda es verdad. Pero la racionalidad es insuficiente para entender todo el quehacer científico. Se ha pensado la ciencia también desde los aspectos sociales e históricos. Esto también es verdad. Pero todo el conocimiento científico arranca de los sentidos, pero entendidos desde el momento de formalidad de realidad más que desde el momento de contenido, como hace el empirismo.

PALABRAS CLAVE: ciencia; filosofía; realidad; logos; razón; Zubiri.

\section{PHILOSOPHY OF SCIENCE AS A RESEARCH PROGRAM IN ZUBIRI}

Copyright: (c) 2016 CSIC. Este es un artículo de acceso abierto distribuido bajo los términos de la licencia Creative Commons Attribution (CC BY) España 3.0.
ABSTRACT: In this paper we attempt to explain the meaning of science according to Zubiri. Although Zubiri did not publish a specific book about science, we attempt to contemplate this topic by following the analysis in three moments of the apprehension of reality that Zubiri makes in his book Sentient Intelligence. Thereafter, we propose that the concept of science must be analyzed in these three moments, the first of which shows that scientific knowledge is rooted in the reality apprehended sentiently. Science is often thought of as a rational activity. This is certainly true, but rationality is insufficient to understand all scientific work. Science has also been considered from social and historical perspectives, which is also true. However, all scientific knowledge starts from the senses, from the moment of formality of reality rather than from the moment of content, as empiricism does. 


\section{INTRODUCCIÓN}

Es de sobra conocido el enorme interés que Zubiri tenía por las ciencias. Se relacionó con ellas de tres modos distintos. En primer lugar, las estudió profundamente en sus más diversas manifestaciones como, por ejemplo, estudió física, biología, lingüística, filología, matemática, etc. En segundo lugar, aunque depurando lo recibido, recibió ciertas influencias de las grandes teorías científicas de su época para su filosofía como, por ejemplo, la teoría de la relatividad en su concepción del movimiento, la genética molecular en su concepción de la esencia, etc. Finalmente, en tercer lugar, intentó pensar filosóficamente qué es la ciencia y cuál es su unidad y diferencia con la filosofía. De este tercer punto, lo que nos interesa destacar aquí es meditar qué es ciencia en Zubiri. Aunque Zubiri no dejó un libro específico sobre el tema, es posible "intentar" reconstruir su pensamiento filosófico en torno a este tema. Esto es lo que queremos delinear aquí, iluminados especialmente desde su gran obra Inteligencia sentiente. Nos proponemos, entonces, dicho en lenguaje zubiriano, una especie de "noología de la ciencia" centrando el tema en averiguar qué es la ciencia en sus diversos niveles de aprehensión de realidad.

Para orientarnos en el tema, hay que señalar que el problema filosófico de la ciencia puede presentarse, al menos, desde tres aspectos intrínsecamente relacionados, no necesariamente excluyentes entre sí. En primer lugar, la ciencia podría pensarse como una actividad "racional" con un "método" propio, distinto, por ejemplo, a la actividad filosófica. Aquí es importante meditar qué papel juega la inducción, el modelo hipotético-deductivo, etc., en corrientes como el positivismo lógico, el falsacionismo de Popper, los programas de investigación de Lakatos, etc. Desde este punto de vista, es perfectamente posible sostener que no todo lo que se ha reconocido como "científico" posee tal carácter, sino sólo aquello que se ha realizado con el modo racional apropiado. Incluso, en un caso extremo, alguien podría sostener que todavía hoy no existe un verdadero conocimiento científico. En segundo lugar, podría pensarse la ciencia como una especie de "interpretación social e histórica" del quehacer científico. Podrá negarse a la ciencia haber encontrado un conocimiento científico verdadero, según el modo racional apropiado, pero lo que es innegable es que existe la ciencia como "hecho" socio-histórico. Es científico aquello que un gremio ha legitimado como tal. Aquí entonces cobra una gran importancia el análisis de la "historia y sociología del conocimiento científico". La ciencia es una actividad no sólo individual sino también, y de forma más eminente, social. En este aspecto destaca Kuhn, Bloor, Barnes, etc. Por último, podría pensarse la ciencia como una especie de "modo de presentación de lo aprehendido". Éste es el momento "radical" donde se instala el pensamiento de Zubiri sobre la ciencia. Para él, ciencia y filosofía estudian la realidad aprehendida sentientemente; desde aquí arranca su esencial unidad y diferencia.

Para entender esto, hay que tener en cuenta el análisis y conceptuación que Zubiri ha hecho en Inteligencia sentiente de lo que es realidad, sentir, inteligir, actualidad, etc. No podemos desarrollar todos estos temas aquí, así que vamos a desarrollar algunos, los más importantes, que pueden perfilar un posible programa de investigación de filosofía de la ciencia en Zubiri. Para este punto es importante atender a la distinción entre los tres modos de intelección sentiente llamados aprehensión primordial, logos y razón. Desde esta distinción es desde donde tenemos que pensar lo que es "ciencia". Este término, en Zubiri, si bien es cierto no es equívoco tampoco es unívoco. "Ciencia" se dice de múltiples maneras. Veamos estos tres momentos brevemente y su relación con lo que es ciencia.

\section{1) CIENCIA Y APREHENSIÓN PRIMORDIAL DE REALIDAD}

El modo primario de percepción de realidad es lo que Zubiri llama "aprehensión primordial de realidad". En ella se actualiza la realidad de forma directa, inmediata y unitaria. En este modo de actualización, para Zubiri, se nos actualiza lo real como algo "solamente" en y por sí mismo. Para ello, en lo percibido nos fijamos o atendemos a lo real en tanto real. Aquí no es posible el error de ningún tipo. El error sólo es posible en los modos ulteriores. Ahora bien, en esta aprehensión se nos muestran dos momentos fundamentales. Por una parte, hay que atender a los "contenidos" presentados. Contenido apunta aquí a lo "presente" en nuestras aprehensiones. Así, aprehendemos colores, sabores, etc. (dejo de lado el problema de si todos los contenidos en tanto tales son propios de este modo de aprehensión). Pero hay algo más. Hay que atender también al "modo" o manera como esos mismos contenidos aprehendidos se presentan en el hombre. Al modo o manera como los contenidos se presentan es a lo que Zubiri llama "formalidad". La filosofía, por lo general, sólo había "atendido" a lo meramente presente (contenido) descuidando que aquello mismo presente percibido se muestra de un cierto modo o manera. Por ello, Zubiri suele decir: "la filosofía se ha fijado solamente sobre el contenido, pero ha resbalado sobre la formalidad" (2006, p. 188). Esta forma- 
lidad es una modalidad dada físicamente, aunque de una manera algo distinta a como se nos dan los contenidos. Por ello dice que la formalidad "es un momento físico de las cosas reales en cuanto sentidas en impresión de realidad. No es algo físico al modo como lo es su contenido, pero es, sin embargo, algo físico: es lo físico de la formalidad, esto es, la física del trans en cuanto tal" (Zubiri, 1980, p. 123).

Ahora bien, en nuestras aprehensiones, el modo como se presentan los contenidos aprehendidos es como "formalidad del de suyo" (Zubiri, 1980, p. 191) o "formalidad de realidad o reidad". La realidad no es una "cosa en sí", independiente de nuestra aprehensión. Algo puede aprehenderse como realidad, aunque sólo una persona lo perciba. Tampoco la realidad se muestra básicamente como elaboración mía. Yo no percibo, por ejemplo, cómo mi cerebro construye el mundo. Esto es algo que la ciencia tendrá que averiguar ulteriormente en otros niveles de intelección. Realidad no es cosa en sí ni cosa en mí, como sostiene Diego Gracia (1986, p. 139). La realidad es algo dado "en" la aprehensión, pero como "de suyo". Tenemos así dos momentos indisolubles; contenido y formalidad. Si consideramos el momento de formalidad de realidad, por sí mismo, esto nos muestra el momento "transcendental". Transcendental no es aquello que está más allá de los contenidos aprehendidos sino que es el momento de formalidad de realidad en tanto "abierto" al contenido. La realidad como formalidad no se agota en los contenidos presentados, pues ser real es "más" que tener tal o cual contenido. Ahora bien, este ser "más" que los contenidos no significa que la realidad sea un "más" sin ellos. No hay "formalidad" de realidad sin "contenidos". Desde este respecto se nos muestran, por ejemplo, las formas y modos de realidad, el momento de respectividad, el carácter mundanal, etc. Éste es precisamente el tema radical de la filosofía, para Zubiri; un estudio de lo real en tanto que real, un estudio de lo transcendental.

Pero esta misma realidad puede ser atendida desde otro respecto. Contenido, decíamos, es aquello "presente". Formalidad de realidad es la manera "de suyo" o "en propio" en que esos mismos contenidos se presentan. Son dos momentos de la misma aprehensión humana. Así, no aprehendemos mero contenido, sino "contenido" real. Si atendemos a lo real por el lado de su contenido, entonces aprehendemos predominantemente lo real desde su aspecto que Zubiri llama "talitativo". Por ello, nos dice que el "contenido en cuanto aprehendido como algo 'de suyo' ya no es mero contenido, sino que es 'tal' realidad. Es lo que llamo talidad" (Zubiri, 1980, p. 121). El contenido es lo que determina que lo real sea de "tal o cual" manera.

Decíamos que, para Zubiri, la filosofía es el estudio de lo real en tanto real o lo que es lo mismo, el estudio de lo transcendental. En cambio, la ciencia estudia lo real en tanto "tal", es decir, ve lo real predominantemente por el lado de los contenidos. La diferencia o criterio de demarcación de la ciencia y la filosofía es, entonces, esencialmente "aspectual", en el sentido en que atienden predominantemente a aspectos distintos de la misma aprehensión de realidad. Ahora bien, como decíamos, hay que tener en cuenta que los contenidos aprehendidos no son algo meramente presente, sino que son sentidos en formalidad de realidad. Uno de los modos de realidad aprehendidos es, para Zubiri, el modo "direccional" Ilamado "hacia". Lo real sensible aprehendido nos remite tanto "hacia" otras cosas reales sentidas como "hacia" su fundamento, "hacia" aquello allende la percepción que determina lo presente. Este modo de realidad como "hacia" no es algo que "pone" el investigador, sino que es un modo sentiente de realidad "dado" en la aprehensión de realidad. Ahora bien, la ciencia consiste, en este nivel de análisis del acto de intelección, en ese "modo de presentación" de lo real llamado "hacia" por el cual estamos remitidos inexorablemente, en última instancia, al fundamento de lo real aprehendido. La ciencia pretende decirnos, para Zubiri, en qué consiste las cualidades o contenidos percibidos, en qué se fundamentan. Por ello, nos dice que "averiguar qué son estas cualidades reales en el mundo allende lo formalmente sentido es justo la obra de la ciencia" (Zubiri, 1980, p. 154). Esta tarea de la ciencia se funda, entonces, en la aprehensión primordial de realidad. Es la realidad misma aprehendida, y no la iniciativa del científico, la que nos arrastra a afirmarla y conocerla. La ciencia es, en este sentido, algo inevitable. De ahí que Zubiri nos diga:

La ciencia no es, pues, una ocurrencia caprichosa, ni una arbitraria complexión de conceptos, sino que es algo inexorable sean cualquiera sus modos. Tanto los modos del 'hacia' del hombre más primitivo como los nuestros propios, son modos de 'ciencia', esto es, modos de una marcha inexorable desde la realidad percibida hacia lo real allende la percepción (1980, pp. 185-186).

La ciencia, entonces, para Zubiri, se origina del "arrastre" de las cosas reales aprehendidas. Son ellas mismas las que nos llevan a conocerlas. La ciencia es entendida aquí, en su sentido más radical, como algo inexorable. Sin embargo, este arrastre es un arrastre "abierto". Hay muchos modos de fundamentar el "ha- 
cia" aprehendido. Uno de ellos, sólo uno de ellos, es la ciencia moderna, pero, como dice la cita, el hombre primitivo también posee ciencia en la medida en que inexorablemente ha tenido que explicarse teoréticamente la realidad percibida en tanto "tal". Desde este nivel, entonces, ciencia no se identifica formalmente, por ejemplo, con la ciencia moderna que viene desde los tiempos de Galileo. La ciencia es conceptuada aquí como el modo en que la realidad aprehendida nos remite necesariamente "hacia" lo real allende la percepción como fundamento de lo real percibido en tanto tal. Por ello, el concepto de ciencia en Zubiri se utiliza de forma mucho más amplia de lo que suele entenderse en filosofía de la ciencia. Ciencia designa aquí un aspecto transcendental de todo lo aprehendido que es fundamento de otros ulteriores conceptos de ciencia. La ciencia, entendida así, no es algo opcional, sino inexorable para el ser humano. Es un momento de la realidad aprehendida.

Desde este sentido radical de lo que es ciencia en Zubiri se podría intentar meditar las diferencias con otras posturas filosóficas, por ejemplo, investigar cómo se origina la ciencia, es decir, cuál es su principio. Existen aquí básicamente dos posturas en filosofía de la ciencia. Por un lado, se podría sostener que la ciencia debe partir desde el dato bruto de los sentidos, base de lo que podríamos llamar una "observación científica". Es la idea básicamente del empirismo y positivismo (cf. Wartofsky, 1981, p. 137). Por otro lado, como la observación parece estar ya mediada por las teorías científicas, se puede postular que la ciencia debe partir más bien desde un juicio universal postulado hipotéticamente como una "conjetura" (cf. Popper, 1983). El pensamiento de Zubiri parece ser relativamente distinto. La ciencia arranca desde la "aprehensión primordial de realidad". Zubiri no es un empirista que parte del dato sensible, porque aquí se confunde contenido "sensible" y modo de presentación "sentiente" como el "hacia". Es probable incluso que el contenido sensible sea algo totalmente construido y no dado. Si esto es cierto, la observación científica, instalada en el contenido, sería algo construido y mediado por la teoría. Pero, sea eso o no verdad, lo que nos está dado sentientemente son sus modalidades (el "hacia", el "ante mí", etc.) presentes en toda aprehensión de realidad. Por otra parte, la ciencia no parte de un mero juicio. Todo juicio se construye "ya" desde la realidad aprehendida sentientemente, realidad que es formalidad, y no mero contenido.

En síntesis, en este nivel de aprehensión primordial, ciencia es básicamente el "modo talitativo de presen- tación de la realidad como hacia". Desde aquí pueden surgir los otros conceptos de ciencia que veremos a continuación.

\section{2) EL LOGOS CIENTíFICO}

Apoyados en esta aprehensión primordial, lo real así aprehendido queda necesariamente abierto para ser actualizado desde otras realidades aprehendidas. La actualización de una realidad en función de otras es lo que Zubiri llama logos sentiente ${ }^{2}$. Este logos sentiente posee dos momentos. Por una parte, hay un momento de distancia, de retracción del contenido de lo real. Se intelige libremente como sería lo real. En esta distanciación, el hombre puede crear perceptos, fictos y conceptos. Por otra parte, con todo ello se "afirma" cómo sería la cosa "en realidad". Se afirma que lo que sería se realiza en lo que es. En este modo de intelección, nosotros nos movemos con cierta "libertad" de cómo vamos a hacer una afirmación, por ejemplo, nosotros elegimos qué aspecto vamos a tomar de la realidad aprehendida, cómo la vamos a concebir, cómo la vamos a afirmar, etc.

Ahora bien, en el caso de la ciencia, habría que investigar qué particularidades posee el logos científico. Tal vez, se podría afirmar que, para Zubiri, es un logos que es básicamente descriptivo, es decir, que trata de describir principalmente los "contenidos" de una cosa real "en función de" otras cosas reales presentes. Este logos científico posee al menos tres caracteres que habrá que explicitar brevemente.

En primer lugar, hay que señalar qué tipo de juicio o enunciado constituye el logos científico. El más importante parece ser el que Zubiri llama juicio proposicional (cf. 1982, pp. 154-158). Los juicios suelen entenderse clásicamente como juicios predicativos de la forma "A es $B^{\prime \prime}$. Los juicios científicos ciertamente pueden ser predicativos (es el caso de una descripción típica), pero son más bien "funcionales", es decir, se trata aquí de describir qué relaciones talitativas hay entre unas variables con otras. Así, por ejemplo, la ciencia nos dice la relación de presión, temperatura y volumen que existe en un gas, la relación de fuerza, masa y aceleración en un cuerpo, etc. En este tipo de juicio, cualquiera de esas variables puede hacer las veces de sujeto de la oración. Aquí la ciencia le quita el carácter sustancial a la realidad, algo que agrada a Zubiri (cf. 2008, pp. 161-162). En ese mismo caso podrían entenderse las "definiciones operacionales" de la ciencia (cf. Hempel, 1973), las cuales definen un concepto en relación a un instrumento de medida como la masa en función de la balanza, la inteligencia en función de un test, etc. 
En segundo lugar, es posible establecer nuevos juicios apoyándose en otros ya existentes. Es lo que ocurre en una "generalización científica", por ejemplo, por inducción. Se puede llegar aquí a juicios universales, tan importantes en ciencia. Aquí es importante considerar el papel del razonamiento lógico. Es importante investigar qué papel juega la lógica en la ciencia. Zubiri dice muy poco sobre su comprensión de la lógica, dándonos sólo leves pinceladas en Inteligencia sentiente. Sin embargo, distingue claramente entre razonamiento lógico y razón, distinción que es importante para entender el conocimiento científico. Para mayor claridad, volveré sobre este punto más abajo cuando se trate de la razón científica.

En tercer lugar, hay que tener en cuenta que en toda intelección de logos se nos actualiza algo real desde la "unidad" de las demás cosas reales, lo que Zubiri llama "campo". Campo no es una cosa real más que se intelige, sino aquello "desde" lo cual inteligimos algo. Es un "medio" de intelección, algo que nos hace ver las cosas de una determinada manera. Es el medio como "luz" o ser de las cosas. Así, Zubiri nos dice:

No es lo mismo inteligir una cosa en cierto modo individualmente por ella misma que inteligirla en un medio social. No es algo que pertenece a lo inteligido, pero es desde luego algo que hace ver lo inteligido de una manera peculiar [...] No es lo mismo inteligir algo en un medio social (general o especial [gremial]) que inteligirlas en un medio religioso. La sociedad en sus diversas formas, la religión, etc., son desde este punto de vista no lo que inteligimos, sino algo que nos hace inteligir las cosas. En diferentes medios se ven las cosas de distinta manera. Por eso digo que el medio es algo esencial a la intelección en todos los órdenes (1982, p. 76).

Desde aquí podría tratar de entenderse el logos científico en su aspecto socio-histórico. Sería válido tratar de entender el quehacer científico desde su medio social e histórico, como lo ha intentado, por ejemplo $\mathrm{Kuhn}^{3}$, con su concepto de paradigma. Sin embargo, no sería esto lo que radicalmente define la ciencia. El logos está apoyado, como vimos, en la aprehensión primordial de realidad. El medio afecta indudablemente al "contenido" de lo descrito, pero no a la formalidad de realidad. Donde alguien sólo describe una marca sobre un aparato, un científico describe cambios de temperatura. La temperatura es aprehendida culturalmente. Sí, esto puede ser verdad, pero falta incorporar en esta descripción el modo "de suyo" de lo aprehendido, la realidad. La "realidad" no se construiría culturalmente, sólo lo sería "lo real" desde su contenido. En este tipo de pensamiento sobre la ciencia se encuentra, tal vez, la filosofía de Heidegger. Para este pensador, "la ciencia es un modo, y además un modo decisivo, como se nos presenta todo lo que es" (2001, p. 33). Este modo o manera de hacérsenos presentes las cosas es un modo que Heidegger llama "técnico". Esto se asemeja al pensamiento de Zubiri, pero al parecer no como aprehensión primordial, sino como campo de intelección. La técnica, como el paradigma, es el horizonte de intelección. Desde aquí se podría intentar realizar una discusión de Zubiri con estos dos pensadores de la ciencia.

\section{3) LA RAZÓN CIENTÍFICA}

Pero hay una modalidad ulterior que se apoya en las dos anteriores. Es la intelección sentiente como "razón". La razón nos actualiza lo real desde su fundamento. ¿Qué es fundamento? Zubiri nos dice que "fundamento es todo aquello que determina desde sí mismo, pero en y por sí mismo, a lo percibido, de suerte que éste es una realización del fundamento en lo fundado mismo" (1983, p. 161). Se busca entonces, desde lo percibido mismo, aquello que determina por qué es como es, la cosa real percibida. Esta búsqueda de fundamento, es para Zubiri, algo determinado por la aprehensión misma de realidad. No es que el hombre se "proponga" buscar fundamento, sino que el carácter fundamental de la realidad nos es dado impresivamente en la realidad como "hacia". Lo que no nos es dado es "cuál" sea el contenido concreto del fundamento de lo real, tarea difícil e inacabable para Zubiri.

Ahora bien, desde este momento tenemos que pensar la ciencia desde su actualidad racional. Para Zubiri, en sentido general, ciencia se define desde su actualidad racional. Así nos lo dice en su conferencia Qué es investigar: "Investigar las notas o caracteres propios de cada orden de cosas reales es justo lo que constituye la investigación científica, lo que constituye las distintas ciencias. Ciencia es investigación de lo que las cosas son en la realidad" (2006, p. 323). En la razón, recordemos, se nos actualiza lo real desde su fundamento. Es algo inexorable que se impone desde lo real mismo aprehendido. Ahora bien, esta apertura de las cosas reales, en el caso de la ciencia, significa que las cosas reales con sus cualidades o contenidos sensibles nos remiten necesariamente a su fundamento. Esto es en lo que consiste el conocimiento. Así, nos dirá Zubiri: "la intelección de algo en su realidad profunda, es decir, la intelección racional, es lo que formalmente constituye el conocimiento" (1983, 
p. 161). Conocimiento es así un modo de intelección, de actualización de lo real. Es algo que viene impuesto por la aprehensión de la realidad misma. No es algo que radicalmente dependa de nuestra iniciativa. Con esto en mente, hay que tener en cuenta dos ideas importantes para entender qué es el conocimiento científico en Zubiri.

1) En primer lugar, ciencia es conocimiento de lo real en tanto "tal" realidad. Este conocimiento científico es esencialmente de dos tipos. Por una parte, hay que explicar "lo percibido en tanto percibido". Se trata de explicar, por ejemplo, cómo surge el verde en la aprehensión. Es la tarea, en general, de la neurociencia. Por otra parte, hay que explicar lo percibido en tanto "tal realidad". Por ejemplo, explicar qué es el verde no en tanto cómo es percibido, sino en tanto verde (longitud de onda, etc.). Así Zubiri nos dice: "Y entonces la ciencia no es ya sólo una explicación de lo percibido, sino una explicación de la realidad entera del cosmos: es la labor ingente de los conceptos, de las leyes y de las teorías científicas" (1980, p. 188).

2) En segundo lugar, la ciencia es, en este nivel de aprehensión racional, un tipo de conocimiento, un modo de actualización de lo real, entre otros posibles. Zubiri no identifica el conocimiento en general con el conocimiento científico. Hay múltiples modos de ir "hacia" el fundamento de lo real. Todo esto hace que el mismo concepto racional de ciencia tenga, a su vez, diversos matices.

a) En primer lugar, decíamos que hay muchas maneras o figuras de fundamentar lo real. Esto es lo que origina que existan diversas "mentalidades". Para Zubiri, "mentalidad es el modo intrínseco y formal de habitud de lanzamiento hacia las cosas reales" (1983, p. 152). Para él, por ejemplo, ciencia y poesía son dos mentalidades, dos modos de conocimiento, entre otros posibles. Aquí, entonces, la ciencia es una mentalidad teorética talitativa que se distingue de otras mentalidades como la poesía y la metafísica.

b) En segundo lugar, hay que considerar que, aún dentro de una misma mentalidad, hay distintas cualificaciones, en nuestro caso, distintos "modos racionales" de hacer ciencia. Por ello, Zubiri nos dice: "Dentro del intrínseco 'hacia' propio de la ciencia, hay modos diversos; no es lo mismo lo que un primitivo sumerio o akkadio entiende por explicar el mundo, que lo que por explicar el mundo entendió un griego, ni lo que entendió un griego por explicar el mundo es idéntico a lo que nosotros entendemos" (1983, p. 151). Hay, así, entonces, una ciencia sumeria, griega, moderna, etc., entendidas como distintas cualificaciones de una misma mentalidad científica. Cuando en Naturaleza, Historia, Dios, Zubiri distingue entre la episteme griega y la ciencia moderna, tal vez, podríamos entender esa distinción ahora desde Inteligencia sentiente como una distinción "dentro" de la mentalidad científica. Así, en ese texto nos decía:

Todo saber físico es saber el porqué de las cosas. No hay conocimiento más que en la medida en que hay un porqué sabido. Desde el momento en que se sabe el porqué, se conoce eo ipso la inexorable necesidad que penetra en la realidad. Pero este porqué, que se sabe, es distinto en la ciencia y en la epistéme.

La necesidad tiene, efectivamente, en la ciencia un sentido sumamente preciso. Saber, por ejemplo, por qué asciende un globo, o por qué se producen los eclipses, o por qué se hiela el agua, significa saber cómo se produce la congelación, la navegación aérea o la interferencia de las proyecciones luminosas de los astros. Saber 'cómo', es esencialmente saber qué cosas deben acontecer para que acontezcan otras. El 'porqué' de la ciencia es siempre un 'cómo' que recae sobre un 'quién'. Cómo y por quién se produce lo que se produce. El que una explicación resulte complicada procede, en efecto, del número de quiénes tengan que intervenir y de cómo hayan de intervenir.

Pero, en cambio, para la epistéme, el problema del 'porqué' es esencialmente el problema de averiguar qué hay en la causa, que cause determinado efecto. No se trata de determinar cómo se producen las cosas: se trata de averiguar cómo tienen que ser las que las producen. No se trata de saber quiénes las producen, sino qué son esos quiénes que las producen. En realidad, tras el porqué, la ciencia busca el cómo; la epistéme, el qué (1987, pp. 106-107).

Como puede apreciarse, tanto en Naturaleza, Historia, Dios como en Inteligencia sentiente Zubiri mantiene que la ciencia, en este nivel de consideración, es la búsqueda del fundamento de lo real, del porqué de las cosas. Pero, dentro de esta búsqueda, existen distintas modalidades como la episteme griega que busca "el qué de las cosas", a diferencia de la ciencia moderna que busca "el cómo funcional".

c) Finalmente, en tercer lugar, hay que considerar que la misma "ciencia moderna" no se ha entendido de la misma manera. No es lo mismo la ciencia de Newton, Einstein, etc., además de que no todos los filósofos entienden lo científico de la misma manera. El objeto de estudio de la filosofía de la ciencia, en general, se entiende predominantemente como estudio 
sobre la "ciencia moderna". El concepto de ciencia en Zubiri, como vimos, es mucho más amplio. Ahora bien, hay que tener en cuenta que en el libro Inteligencia y razón, Zubiri analiza la estructura del conocimiento en general en tres momentos: su objeto, método y encuentro verdadero. Además, para cada uno de esos aspectos del conocimiento, Zubiri define la manera propia en que la ciencia los entiende. Así nos dice que la ciencia es "un conocimiento que quiere revestir la forma de hechos experimentales [su objeto], de método preciso de experimentación, o de fundamentación de verdades comprobables. Esta triple intención es característica de la ciencia" (1983, p. 173). Aquí hay que entender, ante todo, que Zubiri se refiere a la ciencia no como una especial mentalidad frente a otras, sino específicamente a la "ciencia moderna" que fundó, entre otros, Galileo. Es este sentido de ciencia el que Zubiri quiere analizar en su triple estructura de hechos, método y verdad. No es nuestra intención aquí analizar detalladamente estos tres momentos. Tampoco Zubiri los dejó muy desarrollados desde el punto de vista de la ciencia. Sin embargo, convendría destacar estos tres momentos en la medida en que nos permiten entender qué entiende Zubiri por ciencia moderna en relación a otras concepciones de ella.

En primer lugar, Zubiri sostiene que el objeto del conocimiento científico es el llamado "hecho científico" entendido como "la constatación de la realidad aprehendida en función de conceptos previos" (1983, p. 185). No es un hecho puro, por ejemplo, que un termómetro de mercurio nos "dé" la temperatura, sino que constatamos tal temperatura "a la luz" de lo que sabemos, es decir, que un aumento del volumen del mercurio es proporcional al de la temperatura (a presión constante) y, por tanto, nos da tal valor. La ciencia, como veíamos, se funda en un logos donde los así llamados hechos científicos no surgen desde la mera aprehensión primordial de realidad. Por ello, las críticas que se hacen a la observación pura en ciencias parecen ser correctas (Wartofsky 1981; Chalmers, 2000, etc.), pero la "observación científica" y el "hecho científico" constatado no serían, a nuestro juicio, mera aprehensión primordial, sino logos y razón, respectivamente.

En segundo lugar, hay que meditar qué es el método científico. Zubiri piensa que el "método en general" es un abrirse paso hacia el fundamento (cf. 1983, p. 203) que no consiste en un "razonamiento" de ningún tipo. Así, él nos dice que "el método mismo, no consiste en ser un razonamiento. No es el acceso de un juicio verdadero a otro juicio verdadero, porque lo que se busca no es otro juicio sino otra actualización" (1983, p. 207). El razonamiento, la conexión lógica entre juicios no es, para Zubiri, algo que por sí solo consista en una actualización racional. Razonamiento no es lo mismo que razón, es más bien, como vimos, un juicio que se apoya en otros juicios, por tanto, habría que colocarlo en el nivel del logos. Es que, para Zubiri, la razón en general (y con ello la razón científica) es "actualidad" de lo real desde su fundamento. La razón científica nos dice en qué consiste lo real en tanto "tal" realidad, en qué consiste el verde, en qué se funda lo percibido como tal, etc. Nada de esto nos da, por sí solo, la "conclusión" de un razonamiento. Hay algunos ejemplos que pueden ayudarnos a fundamentar lo ya dicho. Por ejemplo, la deducción le parece a Zubiri un razonamiento lógico legítimo, pero no es un método, pues mediante él no actualizamos lo real desde su fundamento. La inducción, por su parte, incluyendo la inducción probabilística, no le parece a Zubiri un razonamiento lógico legítimo, pero sí es un método. Zubiri nos lo explica así:

Nunca se ha conseguido estructurar un razonamiento inductivo. Para ello lo menos que haría falta es que se enunciara lo que suele llamarse el principio de inducción. Y esto, de hecho, no ha sido logrado jamás satisfactoriamente, ni siquiera al recurrir al cálculo de probabilidades para excluir los errores azarosos de la experimentación. Por tanto, de hecho no existe un razonamiento inductivo. En cambio, la inducción existe como método estricto y riguroso. Se parte de lo real actualizado en hechos y se va por repetición (conforme a la ley de los grandes números) desde los resultados experimentales a un enunciado general. Este enunciado enuncia la actualización del fundamento (1983, pp. 208-209).

Es interesante destacar que aunque, para Zubiri, el enunciado universal no se siga por razonamiento lógico inductivo de las premisas singulares, sí considere que tal enunciado universal puede ser usado como un juicio racional, dado que puede actualizarnos el fundamento de lo real. Así, por ejemplo, por la observación de varios cisnes blancos podría inferirse inductivamente que "todos los cisnes son blancos". Esta inferencia inductiva, como vimos para Zubiri, no es correcta. Sin embargo, yo puedo utilizar esta información inductiva para afirmar racionalmente no sólo que todos los cisnes son blancos (esto sería una mera generalización del logos), sino que "ser blanco" es algo que constituye o "fundamenta" a un cisne. Sólo entonces nos encontraríamos ante una afirmación ra- 
cional ${ }^{4}$ científica que, por cierto, tendrá que ulteriormente ser verificada. Desde luego, habría que meditar la real valía de este ejemplo en particular y de la inducción en general como método racional, algo que Zubiri no discute en su libro ${ }^{5}$.

Hay que destacar que esta crítica a la inducción como razonamiento lógico ya la había señalado Karl Popper. Él nos dice así:

Es corriente llamar 'inductiva' a una inferencia cuando pasa de enunciados singulares (llamados, a veces, enunciados (particulares'), tales como descripciones de los resultados de observaciones o experimentos, a enunciados universales, tales como hipótesis o teorías. Ahora bien, desde un punto de vista lógico dista mucho de ser obvio que estemos justificados al inferir enunciados universales partiendo de enunciados singulares, por elevado que sea su número; pues cualquier conclusión que saquemos de este modo corre siempre el riesgo de resultar un día falsa: así, cualquiera que sea el número de ejemplares de cisnes blancos que hayamos observado, no está justificada la conclusión de que todos los cisnes sean blancos (1962, p. 27).

Por ello mismo, Popper propuso en ese libro para la ciencia un tipo de método hipotético-deductivo, lo que se ha llamado "falsacionismo". No se parte de juicios singulares (como el inductivismo), sino de un juicio universal a modo de hipótesis o conjetura. Se diferencia del método deductivo clásico en que este juicio universal debe ser contrastado con la experiencia sensible. No es un juicio verdadero intuido directamente (no es una especie de axioma), sino un juicio establecido como hipótesis o conjetura. Esta hipótesis no puede demostrarse que sea verdadera (en eso se diferencia precisamente del inductivismo), pero sí puede probarse que es falsa al entrar en contradicción con un juicio singular. Ello ocurre porque la única inferencia lógica posible por "deducción" es el paso de un juicio singular falso hacia un juicio universal falso. Así, bastaría con encontrar un cisne que no sea blanco para inferir deductivamente que todos los cisnes no son blancos. Popper nos lo dice así:

Pues éstos [los enunciados universales] no son jamás deducibles de enunciados singulares, pero sí pueden estar en contradicción con estos últimos. En consecuencia, por medio de inferencias puramente deductivas (valiéndose del modus tollens de la lógica clásica) es posible argüir de la verdad de enunciados singulares la falsedad de enunciados universales. Una argumentación de esta índole, que lleva a la falsedad de enunciados universales, es el único tipo de inferencia estrictamente deductiva que se mueve, como si dijéramos, en 'dirección inductiva': esto es, de enunciados singulares a universales (1962, p. 41).

Pero todo esto, aunque Zubiri no lo diga expresamente, tampoco podría constituir un método racional científico. La ciencia moderna, para Zubiri, no consiste radicalmente en una actividad racional que consista en afirmar la veracidad o falsedad de unos juicios a partir de otros. Aquí está el punto que nos permite comparar a Zubiri con Popper. La ciencia no sólo no partiría de una conjetura o hipótesis (sino de la aprehensión primordial y logos, como ya vimos) sino que, además, su actividad racional no consiste en la mera falsación de esta conjetura, lo cual se realiza, según Popper, por un razonamiento deductivo, que va de un juicio singular falso (verificado empíricamente) a un juicio universal falso, la hipótesis. Para que esta operación sirva como método científico, como vimos, se requiere algo más: que el juicio universal actualice positivamente a lo real ya aprehendido desde su fundamento. La razón científica tiene fundamento: es la realidad "ya" aprehendida. No hay, en este sentido, an-arquía (cf. Feyerabend, 1981).

En tercer lugar, la actualización racional es algo que admite diversos grados de "verdad racional" entendida como "verificación". Los juicios "racionales" científicos de Zubiri, a diferencia de los puramente lógicos, no son simplemente verdaderos (por inducción) o falsos (por deducción), sino que muestran infinitos matices de actualidad de verdad como "verificación". La verdad racional, que Zubiri llama verificación, es un "ir encontrando" en la historia diversas actualidades del fundamento de lo real. No es algo que sea simplemente verdadero o falso, propio de una verdad lógica de los enunciados. La verdad, para Zubiri, es radicalmente un momento de la "realidad actualizada" más que de los enunciados o juicios.

\section{CONCLUSIÓN}

En suma, el concepto de "ciencia", para Zubiri, se dice de muchas maneras, siendo propio de todo modo de intelección y no de una sola de sus modalidades. Así, ante todo, ciencia atiende al momento talitativo de lo real, al momento predominantemente de contenido. Pero este momento no es estático, sino que, por presentarse los contenidos en el modo de realidad como "hacia", esto nos abre inexorablemente a describir y conocer lo real en tanto tal. Por ello, la ciencia, como veíamos, no es algo caprichoso o fruto de la genialidad moderna, sino algo inexorable. Es 
la ciencia entendida desde la aprehensión primordial de realidad. Pero, por ello mismo, la ciencia debe ser también logos científico. Aquí hay muchas maneras de afirmar, siempre desde un ámbito de libertad. La ciencia moderna, por ejemplo, ha utilizado predominantemente el logos nominal constructo. Finalmente, la ciencia es la investigación de lo que algo es "en la realidad". Es la ciencia como actualidad racional. Aquí hay, a su vez, tres matices: la ciencia como mentalidad frente a otras mentalidades como la poética, la ciencia como cualificación de esa mentalidad (por ejemplo, la ciencia moderna frente al modo de hacer ciencia de la episteme griega) y las diversas concepciones dentro de la misma ciencia moderna ${ }^{6}$.
Por último, cabría seguir meditando la ciencia desde un último modo de actualización estudiado levemente en Inteligencia sentiente; el entendimiento. Se ha estudiado muy poco este modo fundamental de la intelección. Se nos actualiza aquí lo real a la luz de lo ya actualizado en el logos y la razón. Por ejemplo, Zubiri nos dice que un modo de "comprender" es la "explicación causal" en contraposición a otros modos como la interpretación y la funcionalidad personal (cf. 1983, p. 339). ¿Será éste el modo propio de entendimiento de la ciencia?

\section{AGRADECIMIENTOS}

Este artículo es parte del Proyecto Fondecyt Regular $N^{\circ} 1140922$ llamado "Ciencia y realidad en Zubiri".

\section{NOTAS}

1. Dr. Esteban Vargas Abarzúa es Profesor del Instituto de Ciencias Religiosas de la Pontificia Universidad Católica de Valparaíso. Es miembro del Comité Editorial de las Obras de Xavier Zubiri de la Fundación Zubiri de Madrid.

2. La distinción entre la aprehensión primordial y el logos es bastante difícil de establecer y, desde luego, se trata de una distinción que no es una escisión radical, sino una separación de "momentos" dentro de la aprehensión de realidad humana. Es un tema muy extenso que no podemos tratar aquí.

3. Kuhn intenta mirar la ciencia principalmente desde el medio social, desde la comunidad científica. Por ello, nos dice: "No hay un algoritmo neutral para la elección de teorías, no hay ningún procedimiento sistemático de decisión que, aplicado adecuadamente, deba conducir a cada individuo del grupo a la misma decisión. En este sentido es la comunidad de los especialistas, que no sus miembros individuales, la que hace efectiva la decisión" (1993, pp. 304 305).

4. La afirmación racional, para Zubiri, surge de un momento creativo en que esbozamos como tendría que ser el fundamento de lo real. Los resultados de la inducción, en un proceso creativo ulterior, pueden ser usados, como vimos, para afirmar racionalmente lo real, pero tendrán que demostrar su valía, su verificabilidad. La inducción, usada por sí sola como método científico, no sólo falla por ser un razonamiento lógico ilegítimo, sino por no ofrecer, por sí sola, nuevas actualidades racionales de lo real. La inducción es, en general, una generalización de lo ya dado. Ésta es la crítica que Zubiri hace al positivismo lógico: “El positivismo [lógico] sólo es una conceptuación -y no completa- de la intelección campal, pero es ciego para la intelección mundanal, cuyo carácter estructural esencial es la direccionalidad [...] El positivismo lógico es ciego para esta dimensión creadora del conocimiento. Porque crear no es enunciar nuevas proposiciones sino descubrir nuevas direcciones de marcha intelectiva" (1983, p. 216)

5. No parece algo fácil utilizar el resultado de una inducción como fundamento racional. Por ejemplo, si yo encuentro inductivamente una ley que relaciona la masa corporal y la tasa metabólica (por ejemplo, la ley de 3/4 de Kleiber) ¿cómo podría actualizarse esa ley como fundamento? ¿Puede esa ley encontrada darme la "razón" de tales relaciones?
Eso no es claro. De hecho se ha intentado dar "razón", por ejemplo, de la ley de $3 / 4$ de Kleiber, explicando por qué se obtiene este número y no otro, lo que demuestra que tal ley, por sí sola, es difícil que pueda actualizar por sí misma el fundamento racional de lo aprehendido.

6. Habría que meditar si la ciencia moderna consiste en el "hacia" zubiriano racional con su triple estructura de hecho, método y verificación científica. Podría entenderse también como el "todo sirve" de Feyerabend. Por ello nos dice: "Resulta claro, pues, que la idea de un método fijo, o la idea de una teoría fija de la racionalidad, descansa sobre una concepción excesivamente ingenua del hombre y de su contorno social. A quienes consideren el rico material que proporciona la historia, y no intenten empobrecerlo para dar satisfacción a sus más bajos instintos y a su deseo de seguridad intelectual con el pretexto de claridad, precisión, 'objetividad', 'verdad', a esas personas les parecerá que sólo hay un principio que puede defenderse bajo cualquier circunstancia y en todas las etapas del desarrollo humano. Me refiero al principio todo sirve" (Feyerabend, 1981, p. 12). Es algo que habría que meditar. 


\section{BIBLIOGRAFÍA}

Chalmers, A. (2000). ¿Qué es esa cosa Ilamada ciencia? (3a ed.). Madrid: Siglo XXI.

Feyerabend, P. (1981). Tratado contra el método. Madrid: Tecnos.

Gracia, D. (1986). Voluntad de verdad. Para leer a Zubiri. Madrid: Labor.

Heidegger, M. (2001). Ciencia y meditación. En Heidegger, M. Conferencias y artí culos. Barcelona: Del Serbal.

Hempel, C. (1973). Filosofía de la ciencia natural. Madrid: Alianza.

Kuhn, T. (1993). La estructura de las revoluciones científicas. Santiago: Fondo de Cultura Económica.
Popper, K. (1962). La lógica de la investigación científica. Madrid: Tecnos.

Popper, K. (1983). Conjeturas y refutaciones. Barcelona: Paidós.

Zubiri, X. (1980). Inteligencia sentiente, primera parte: Inteligencia y realidad. Madrid: Alianza.

Zubiri, X. (1982). Inteligencia sentiente, segunda parte: Inteligencia y logos. Madrid: Alianza.

Zubiri, X. (1983). Inteligencia sentiente, tercera parte: Inteligencia y razón. Madrid: Alianza.
Zubiri, X. (1987). Naturaleza, Historia, Dios. Madrid: Alianza.

Zubiri, X. (2006). Escritos menores. Madrid: Alianza.

Zubiri, X. (2008): Sobre la esencia. Nueva edición. Madrid: Alianza.

Wartofsky, M. (1981). Introducción a la filosofía de la ciencia. Madrid: Alianza. 